\title{
Protocolo Protésico de Carga Inmediata en Mandíbula y Maxilares Desdentados Utilizando una Cubeta Multifuncional
}

\author{
Protocol Immediately Loaded Prosthesis in Edentulous Jaws Using a Multifunctional Tray
}

Yerko Leighton Fuentealba* \& Juan Carlos Carvajal Herrera**

LEIGHTON, F. Y. \& CARVAJAL, H. J. C. Protocolo protésico de carga inmediata en maxilares desdentados utilizando una cubeta multifuncional. Int. J. Odontostomat., 7(2):299-304, 2013.

RESUMEN: El objetivo del presente trabajo de investigación es la descripción del protocolo para la rehabilitación protésica implanto asistida de carga inmediata, en sujetos desdentados completos maxilares o mandibulares utilizando una "Cubeta protésica Multifuncional Uchile". El protocolo fue desarrollado en un grupo de 96 sujetos desdentados completos mandibulares y/o maxilares, distribuidos en 42 hombres y 54 mujeres con un promedio de edad de 61.5 años, a los cuales se les efectúa un tratamiento protésico implanto asistido, bajo la modalidad de prótesis hibrida en distribución poligonal de arco completo instalada dentro de las $48 \mathrm{hrs}$. siguientes a la inserción de los implantes, tomado una impresión post quirúrgica para la confección de la prótesis de carga inmediata mediante una cubeta protésica multifuncional Uchile. Terminada la cirugía de inserción de implantes, se verifican los Torques quirúrgicos de inserción implantario y los valores de cuociente de estabilidad implantaria (ISQ), procediendo a la toma inmediata de una impresión mediante la técnica de cubeta multifuncional UChile, la cual permite registrara , la altura facial inferior del rostro, el posicionamiento de los implantes, el posicionamiento de la topografía gingival y su máxima intercuspidación. Se envía al laboratorio dental para la confección de una prótesis hibrida que incluye una barra rígida de titanio de sección circular de $3 \mathrm{~mm}$. de diámetro soldada ferulizando los implantes. Durante la inserción protésica se verifica el ajuste pasivo, clínico y radiográfico de la estructura metálica, se efectúa el control oclusal y el control del contorno protésico estético. Se instalaron un total de 96 prótesis de carga inmediata sobre 436 implantes con un torque de inserción quirúrgico promedio de $40.45 \mathrm{~N}$, y un valor promedio de ISQ de 58.6. No se registraron secciones de barra por desajustes, sólo se efectuaron ajustes oclusales menores y eliminación de sobre contornos protésicos. La rehabilitación protésica implanto asistida de carga inmediata en sujetos desdentados completos, utilizando la cubeta Protésica multifuncional UChile, en maxila o mandíbula desdentada, presenta un resultado clínico predecible y reproducible, observando exactitud y pasividad de la estructura protésica a instalar .

PALABRAS CLAVE: carga inmediata, prótesis de carga inmediata.

\section{INTRODUCCIÓN}

La pérdida o ausencia de los dientes afecta al individuo desde la perspectiva física, social y psicológica, especialmente en el ámbito de la autoestima, por lo tanto, la reconstrucción dentaria de pacientes total o parcialmente desdentados, cobra una gran importancia desde las perspectiva biosicosocial de salud (Felce \& Perry, 1995)

La introducción por Branemark et al., (1969, 1977) en el año 1977 del concepto y de los principios de la óseointegración, describiendo un protocolo de tratamiento protésico conocido como "Protocolo Bränemark", proporcionó a los dentistas una nueva herramienta en el tratamiento del edentulismo, los que tradicionalmente habían sido tratados mediante las modalidades de prótesis removibles y/ o prótesis fijas, soportadas por los dientes remanentes.

Las modalidades de tratamiento protésico a través de implantes dentales contemplan la instala-

* Profesor Asistente, Facultad de Odontología, Universidad de Chile. Académico del programa de Especialización Profesional en Rehabilitación Oral y Programa de Especialización profesional en Implantología BucoMáxiloFacial.

** Profesor Asociado del Departamento de Prótesis, Facultad de Odontología, Universidad de Chile. Director del programa de Especialización profesional en Rehabilitación Oral y Director de Prótesis del programa de Especialización profesional en Implantología BucoMáxiloFacial. 
ción diferida en el tiempo de la prótesis, con relación a la inserción implantaria o la modalidad de instalación inmediata de implantes y prótesis, acortando esta última, los tiempos finales del tratamiento protésico, otorgando un mayor beneficio a los pacientes (Adell et al., 1981; Brånemark et al., 1995).

La carga inmediata, la carga funcional inmediata, la función oclusal inmediata o carga oclusal funcional a la luz de la evidencia clínica y científica disponible, es una modalidad terapéutica predecible, con altas tasas de éxito y sobrevida para los implantes y las prótesis. Su utilización como protocolo quirúrgico protésico de elección, estará condicionada a la evaluación cuidadosa de ciertas consideraciones que deben ser conocidas y reconocidas por el dentista, durante la etapa de planificación de tratamiento, para así ofrecer de la manera más eficaz, una modalidad terapéutica que acorte efectivamente los tiempos de la rehabilitación y a la vez, brinde un mayor confort físico, mental y social (Balshi \& Wolfinger, 2003; Parel, 2001; Parr et al., 1993; Brånemark et al., 1999; van Steenberghe et al., 2002).

La carga inmediata es una modalidad protésica que se sustenta en la comprensión de las respuestas tisulares de los tejidos duros y blandos, referidas a procesos reparativos y cicatrizales, cuando los implantes son sometidos a una carga oclusal directa. Los principios fundamentales en que se sustentan dichas respuestas tisulares, a fin de constribuir al éxito de la óseointegración son la estabilidad primaria de los implantes, determinada por la densidad ósea del lecho donde se aloja, la técnica quirúrgica a utilizar y el diseño. La ferulización rigida representa un objetivo primordial, a fin de limitar el micro-movimientos en la interface hueso-implante, durante la función masticatoria.por otro lado el ajuste pasivo de la prótesis sobre las plataformas implantarias, evita las tensiones y micromovimientos del pilar protesico evitando asi tranferir los micromovimientos al implante.

\section{MATERIAL Y MÉTODO}

El protocolo fue desarrollado en los programas de Rehabilitación Oral e Implantología Buco MáxiloFacial de la Universidad de Chile, enfocado en los pacientes desdentados totales candidatos a recibir una prótesis de carga inmediata maxilar o mandibular. Se incluyeron todos los pacientes desdentados totales que consultaron por libre demanda en la clínica de especialidades de la Escuela de Graduados, entre abril de 2009 a diciembre de 2010, los cuales fueron informados respecto de las condiciones de tratamiento que se utilizarían para resolver su situación clínica de desdentamiento total. Se obtubo su consentimiento informado y fueron los pacientes fueron reclutados para aplicar el protocolo bajo los siguentes criterios de inclusión

Criterios de inclusión. Pacientes desdentados completo maxilar o mandibular, con hueso cicatrizado.

Hombres o mujeres mayores de 18 años.

Torque mínimo de inserción sobre $32 \mathrm{Ncm}$ en todos sus implantes instalados

Mínimo de 4 implantes en distribución poligonal

Ausencia de enfermedad sistémica que contraindique un tratamiento de protésico implantoasistida

Criterios de exclusión. Pacientes irradiados en la cabeza y el cuello dentro de 12 meses antes de la cirugía.

Presencia del hábito de fumar.

Presencia de enfermedad periodontal no tratada, en la arcada antagonista.

\section{El protocolo es dividió en tres fases.}

-Fase Pre operatoria: El protocolo comienza con la toma de impresiones de Alginato de maxilar y mandíbula para la obtención de modelos de estudio, los cuales fueron posicionados en un articulador semi-ajustable tipo Whip Mix, registrando las relaciones máxilo mandibulares a través de rodetes de altura. Se realiza la selección del color y molde dentario confeccionando un enfilado dentario de prueba, el que una vez aprobado por el paciente se envía a acrilizado protésico en resina transparente. Luego es probado en boca, ajustado y estabilizado con un tope de mordida anterior y dos topes posteriores en acrílico de auto curado, en una posición de máxima intercuspidación (MIC). Se incluyen en el marcadores radiopacos en los sitios implantario de acuerdo a las necesidades biomecánicas protésicas implantarias, conformando así una guía radiológica, para la toma de un scanner dental con la guía posicionada en MIC. Confirmado los sitios implantarios, esta Guía Imagenológica podría será utilizada también, como una Guía Quirúrgica al momento de insertar los implantes (Fig. 1).

Fase de toma de impresión con la cubeta multifuncional. Insertados los implantes, se verifica y se registra el valor de Torque de progresivo Inserción el cual debe ser superior a $35 \mathrm{Ncm}$ para todos los 


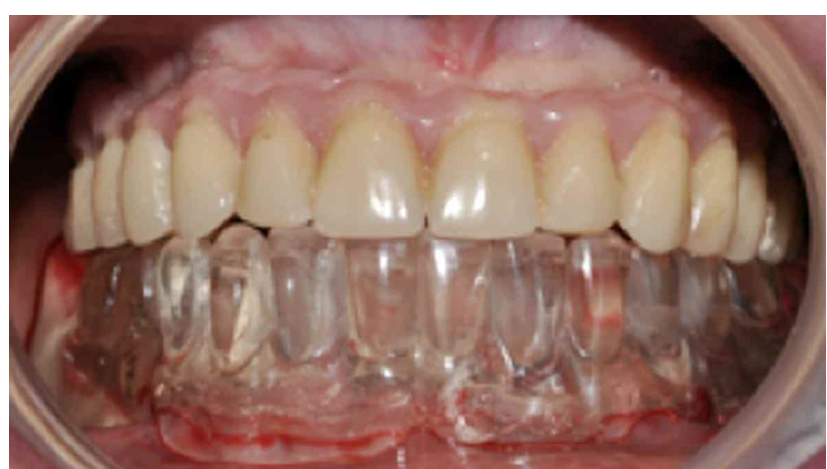

Fig. 1. Prueba de Cubeta protésica Multifuncional UChile.

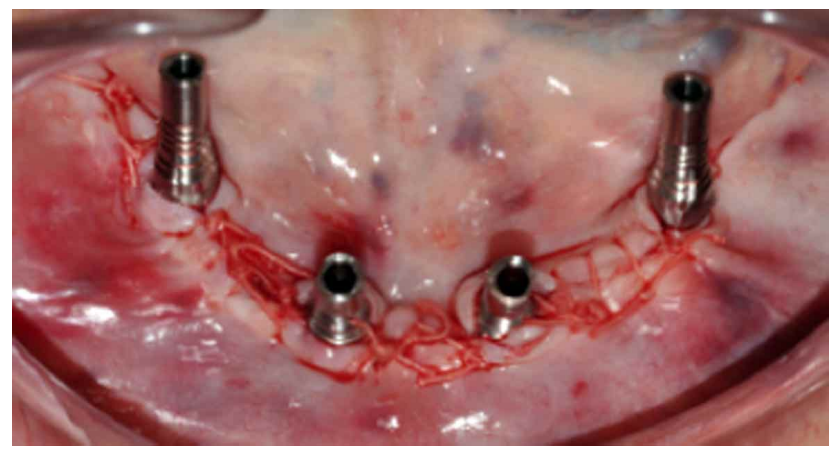

Fig. 2. Pilares protésico instalados y suturada la herida.

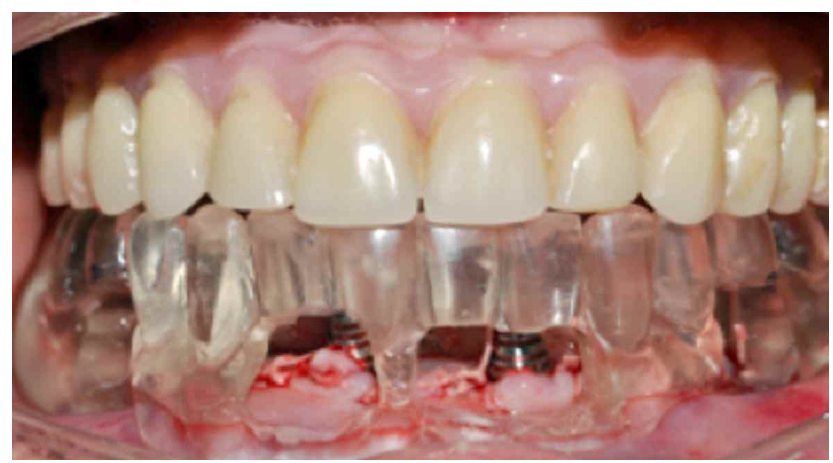

Fig. 3. Cubeta multifuncional estabilizada sobre la mucosa del reborde alveolar, ahuecada y definida sus ventanas vestibulares frente a cada uno de los pilares de titanio, en posición de máxima intercuspidación, preparada para fijar el conjunto cubeta multifuncional / pilares temporales de titanio, con resina acrílica de autopolimerización.

implantes instalados, luego se posicionan los pilares protésicos, los cuales son verificados en sus posición con una radiografia digital intra quirurgica. Para finalizar se procede a suturar el colgajo (Fig. 2).

Fuera del pabellon se procede a realizar perforaciones en sentido oclusal para permitir el paso de la cubeta multifuncional a travéz de los pilares protésicos instalados en boca, verificando el correcto asentamiento de la cubeta sobre la mucosa del reborde alveolar.
Logrado el libre asentamiento de la cubeta multifuncional sin que los pilares toquen la guia, lo cual modificaria la poscion pre establecida, se verificando la oclusion dentaria, la altura facial inferior del rostro a travéz de los topes de oclusión anterior y posteriores, se procede a realizar en la cubeta multifuncional Uchile ventanas vestibulares que permitan acceder a los pilares temporales de titanio ubicados sobre los implantes (Fig. 3).

Encontrándose el paciente en MIC se une mediante resina acrílica de autopolimerización la cubeta multifuncional a cada uno de los pilaresde titanio a travez de las ventanas vestibulares, uno a la vez, se espera la polimerización. Verificada la unión rígida entre los pilares protesicos y la cubeta multifuncional, se solicita al paciente abrir la boca para reforzar la union de los pilares protesico con la cubeta por lingual (Fig. 4).

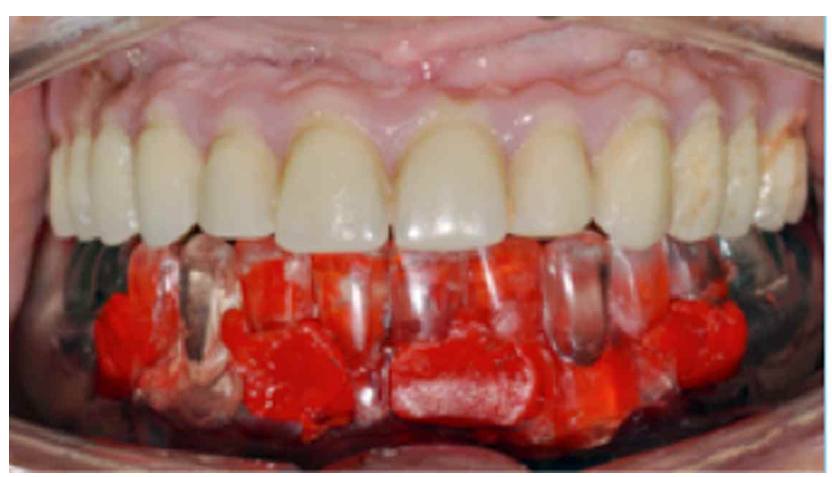

Fig. 4. Cubeta multifuncional fijada a sus pilares protésicos y con sus topes oclusales en MIC.

Para finalizar, siempre que el espacio lo permita, se injecta silicona liviana entre la cubeta y la encía, con el fin de registrar mejor la nueva topografia gingival, se retiran los tornillos prótesicos y se demonta el conjunto pilares temporales de titanio y cubeta multifuncional Uchile (Fig. 5), se lava, y se posicionan los análogos de implante, los cuales son ferulizados entre ellos mediate un segmento de alambre de media caña y acrilico, dejando una estructura rigida entre ellos.

Se envía al laboratorio dental solicitando una prótesis híbrida, con una barra de titanio soldada de sección circular de $3 \mathrm{~mm}$. uniendo todos los implantes a travéz de los pilares protésicos, para ser instalada en $48 \mathrm{~h}$.

Fase de carga inmediata. La prótesis terminada es evaluada en su modelo antes de ser instala en boca, 


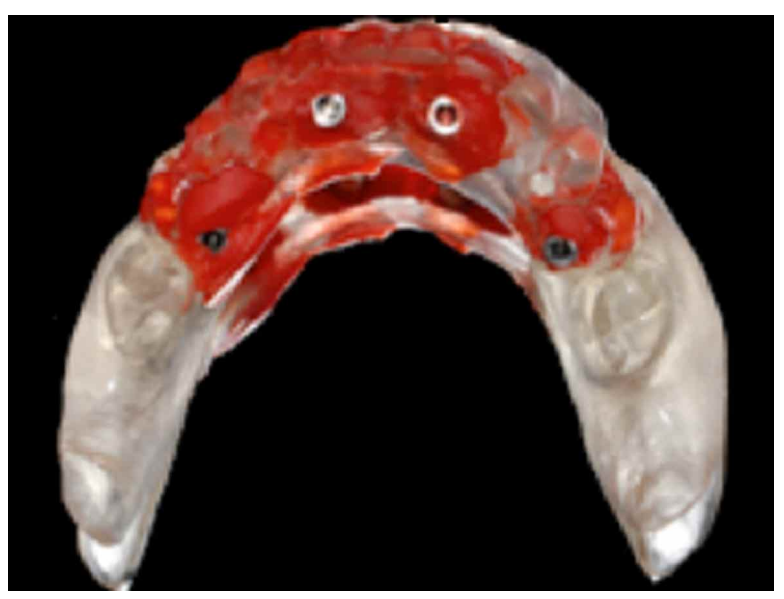

Fig. 5. Conjunto Cubeta multifuncional / pilares de titanio ferulizados con resina acrílica desmontada de boca.

se verifica pasibidad y sobrecontornos de los elementos estéticos, luego se inserta en boca verificando su asentamiento y ajuste pasivo, se verifica la oclusión, sobrecontornos y se realiza un control radiográfico de asentamiento protésico (Fig. 6).

Se realizan los ajustes y el paciente entra en la fase de control protésico (Fig. 7).

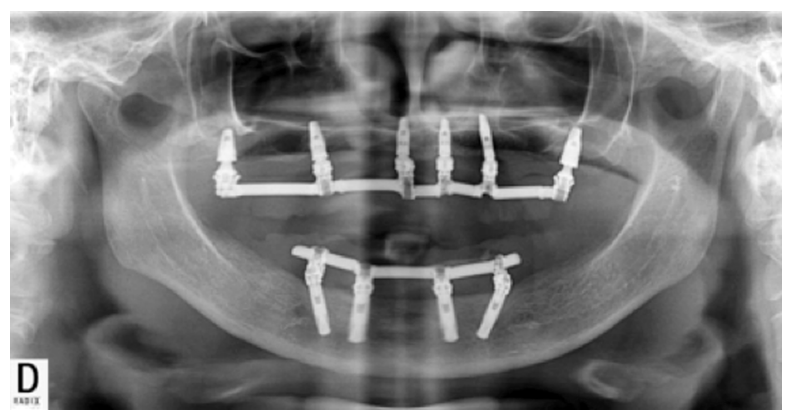

Fig. 6. Radiografia panorámica de control de implantes y barra de titanio inferior para su protesis de carga imnediata.

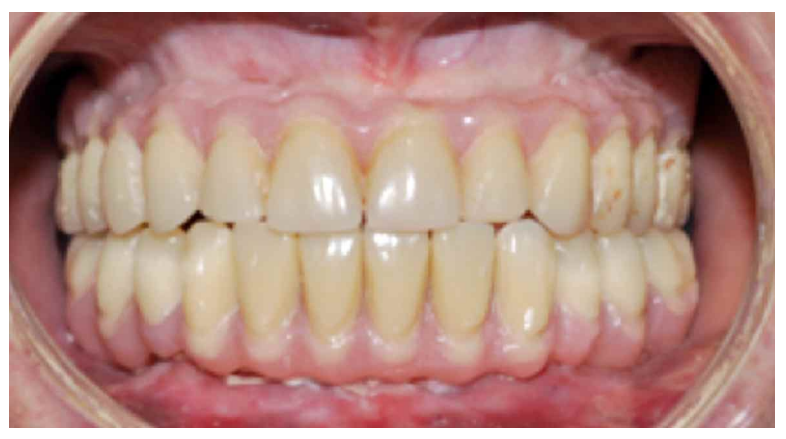

Fig. 7. Prótesis de carga inmediata instalada.

\section{RESULTADOS}

El protocolo fue desarrollado en un grupo de 96 sujetos desdentados completos mandibulares y/o maxilares, distribuidos en 42 hombres y 54 mujeres con un promedio de edad de 61,5 años, a los cuales se les instalaron un total de 436 implante (Tabla I). los valores de torque de inserción progresivo promedio fueron de $40,45 \mathrm{~N}$, y un valor promedio de ISQ de 58,6. Se confeccionaron un total de 96 prótesis hibridas con 96 barras metálicas soldadas en titanio de sección circular, a las cuales sólo se efectuaron ajustes oclusales menores (Fig. 8) y eliminación de sobre contornos protésicos (Fig. 9), no se realizo ningún corte de las barras soldadas y todas las prótesis fueron insertadas a las $48 \mathrm{~h}$ de realizada la cirugía.

Tabla I. Distribución de implantes según modalidad quirúrgica.

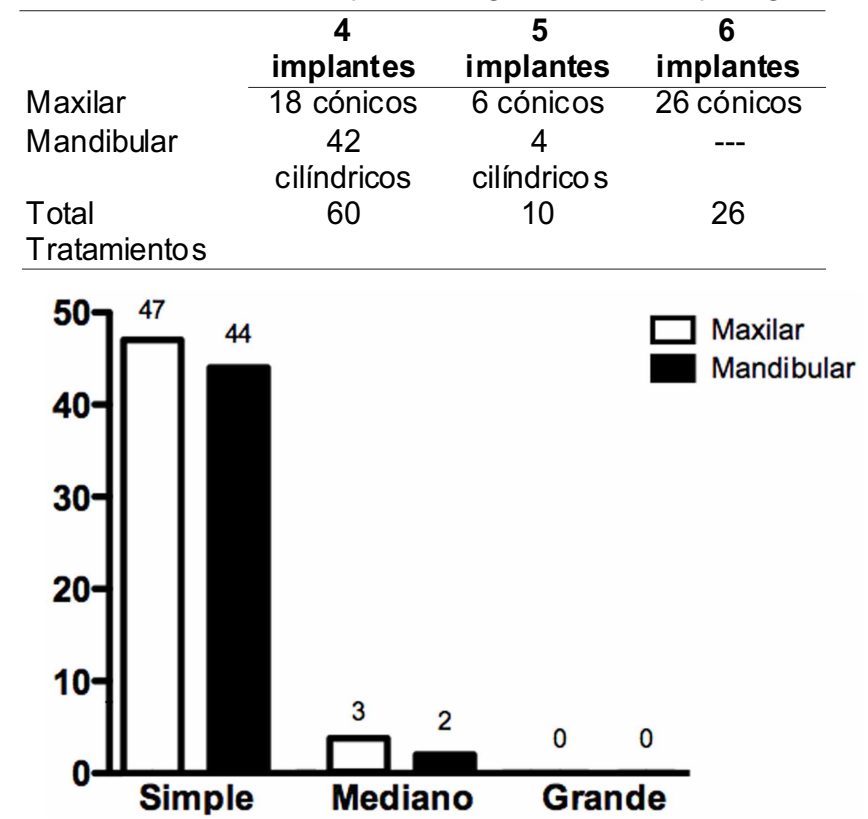

Fig. 8. Distribución de desgastes a los elementos estéticos dentarios.

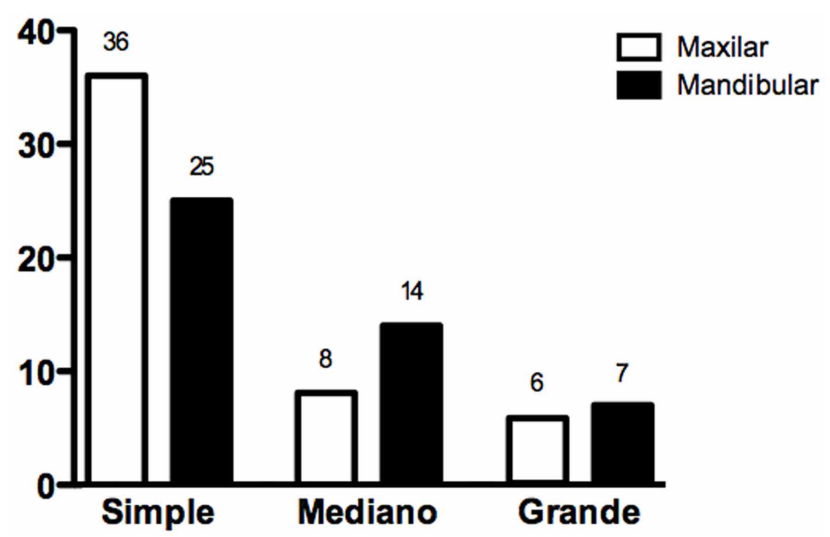

Fig. 9. Distribución de desgastes a los elementos estéticos de encía artificial. 


\section{DISCUSIÓN}

Un problema protésico habitual de los protocolos de carga inmediata es la falta de rigidez de la estructura protésica, tal como describió Maló et al., (2005) en la fractura de los elementos acrílicos, de su protocolo de carga protésica basado en la confección de una prótesis completamente acrílica sobre cuatro pilares de titanio, lo cual trae como complicación un posible fractura de los acrílicos y pone en riesgo la estabilidad primaria del implante con la consecuente molestia para el paciente y los cosos asociados al sumar citas clínicas (Misch et al., 2004: Fornés et al., 2007).

La importancia de incluir una estructura soldada rígida en la prótesis de carga inmediata están basados en los momentos iniciales de la inserción de los implantes donde no existe una conexión celular de la superficie del implante con el hueso y la aposición de hueso solo tiene lugar en la segunda semana de insertado el implante, pero no tiene la capacidad de retención creada por el termino del proceso de oseointegración. Es así como la estabilidad inicial otorgada por el torque quirúrgico de inserción es el responsable de mantener la estructura en su lugar.

El mayor riesgo de sobrecarga oclusal ocurre aproximadamente entre la tercera a la quinta semana de oseointegración donde la interface ósea está poco mineralizada y desorganizada pudiendo incrementar la movilidad implantaría por sobre los márgenes fisiológicos llevando al implante a sufrir una ausencia de la oseointegración (Widmann \& Bale, 2006; Jaffin et al., 2004). Por lo tanto resulta imprescindible que la prótesis de carga inmediata realizada debe ferulizar rígidamente a todos los implantes para evitar la incidencia de micromovimientos superiores a 150 micrometros los cuales interfieren negativamente en la fase cicatrización hueso-titanio. La ferulización protésica inmediata unida a la estabilidad primaria sobre los $32 \mathrm{~N}$ de inserción quirúrgica de los implantes debe asegurar el éxito del tratamiento implantológico (Widmann \& Bale; Jaffin et al.).

El diseño protésico para la carga inmediata debe minimizar las fuerzas oclusales no axiales que no son favorables para el éxito del tratamiento y facilitar la disipación de estas a lo largo de toda sus estructura rígida metálica. En este sentido, una recomendación importante para favorecer la oseointegración durante la rehabilitación protésica de carga inmediata es en lo posible evitar la realización de los extremos libres (cantilevers) o bien minimizar los extremos, de esta forma se disminuir complicaciones como la fractura de la barra o bien el aflojamientos de los tornillos y agregar al diseño una estructura rígida soldada a los pilares protésicos (Misch \& Bidez, 2005; Wood et al., 2004; Taylor et al., 2000).

\section{CONCLUSIONES}

Se sugiere que la rehabilitación protésica implanto asistida de carga inmediata, en maxilares o mandíbulas de sujetos desdentados completos, utilizando la "Cubeta Multifuncional UChile", presenta un resultado clínico predecible y reproducible, observando exactitud y pasividad de la estructura protésica.

LEIGHTON, F. Y. \& CARVAJAL, H. J. C. Protocol immediately loaded prosthesis in edentulous jaws using a multifunctional tray. Int. J. Odontostomat., 7(2):299-304, 2013.

ABSTRACT: The purpose of this research is the description of the protocol for implant-assisted prosthetic rehabilitation immediately loaded in edentulous subjects maxillary or mandibular complete using a "Multifunctional tray Prosthetic Uchile". The protocol was developed in a group of 96 subjects complete edentulous mandible and / or maxilla, distributed in 42 men and 54 women with an average age of 61.5 years, which makes them assisted implant prosthetic treatment, the form of distribution polygonal hybrid prosthesis in full arch installed within $48 \mathrm{hrs}$. after insertion of the implant an impression post surgical taken for making the prosthesis immediately loaded a multifunctional tray prosthetic Uchile. After surgery the implant insertion, the torques are verified surgical implant insertion quotient values and implant stability (ISQ), proceeding to the immediate seizure of a print by UChile multifunctional tray technique, which allows recorded, the lower face facial height, positioning of the implants, the positioning of the gingival topography and its maximum intercuspation. It is sent to the dental lab for the fabrication of a hybrid prosthesis including a rigid bar titanium circular section of $3 \mathrm{~mm}$. splinting welded diameter implants. During insertion prosthetic passive fit is verified, clinical and radiographic metal structure, control is effected occlusal and prosthetic contour control estético. Installed a total of 96 immediately loaded prosthesis on implants with a torque 436 Average surgical insertion of $40.45 \mathrm{~N}$, and an average value of 58.6 ISQ. There were no bar sections by mismatches, only minor adjustments were made occlusal and prosthetic removal on contours. The implant-assisted prosthetic rehabilitation of edentulous subjects immediate loading in full, using the multipurpose tray UChile Prosthetics in edentulous maxilla or mandible presents a predictable and reproducible clinical outcome, noting accuracy and passivity of the prosthetic structure installed.

KEY WORDS: inmediated loading, immediately loaded prosthesis. 


\section{REFERENCIAS BIBLIOGRÁFICAS}

Adell, R.; Lekholm, U.; Rockler, B. \& Brånemark, P. I. A 15year study of osseointegrated implants in the treatment of the edentulous jaw. Int. J. Oral Surg., 10(6):387-416, 1981.

Balshi, T. J. \& Wolfinger, G. J. Immediate loading of dental implants in the edentulous maxilla: case study of a unique protocol. Int. J. Periodontics Restorative Dent., 23(1):3745, 2003.

Brånemark, P. I.; Adell, R.; Breine, U.; Hansson, B. O.; Lindström, J. \& Ohlsson, A. Intra-osseous anchorage of dental prostheses. I. Experimental studies. Scand. J. Plast. Reconstr. Surg., 3(2):81-100, 1969.

Brånemark, P. I.; Engstrand, P.; Ohrnell, L. O.; Gröndahl, K.; Nilsson, P.; Hagberg, K.; et al. Brånemark Novum: a new treatment concept for rehabilitation of the edentulous mandible. Preliminary results from a prospective clinical follow-up study. Clin. Implant Dent. Relat. Res., 1(1):216, 1999.

Brånemark, P. I.; Hansson, B. O.; Adell, R.; Breine, U.; Lindström, J.; Hallén, O.; et al. Osseointegrated implants in the treatment of the edentulous jaw. Experience from a 10 -year period. Scand. J. Plast. Reconstr. Surg. Suppl., 16:1-132, 1977.

Brånemark, P. I.; Svensson, B. \& van Steenberghe, D. Tenyear survival rates of fixed prostheses on four or six implants ad modum Brånemark in full edentulism. Clin. Oral Implants Res., 6(4):227-31, 1995.

Felce, D. \& Perry, J. Quality of life: its definition and measurement. Res. Dev. Disabil., 16(1):51-74, 1995.

Fornés, E.; Velasco, E.; Ortega, F.; García, A. \& Segura, J. J. La carga inmediata con implantes Microdent en el maxilar supe- rior. II. Aspectos prostodóncicos. Av. Periodon., 19(Supl. 1):17-23, 2007.

Jaffin, R. A.; Kumar, A. \& Berman, C. L. Immediate loading of dental implants in the completely edentulous maxilla: a clinical report. Int. J. Oral Maxillofac. Implants, 19(5):721-30, 2004.

Maló, P.; Rangert, B. \& Nobre, M. All-on-4 immediate-function concept with Brånemark System implants for completely edentulous maxillae: a 1-year retrospective clinical study. Clin. Implant Dent. Relat. Res., 7 (Suppl. 1):S88-94, 2005.

Misch, C. \& Bidez, M. W. Occlusal considerations for implantsupported prosthesis: implant-protected occlusion. En: Misch, C. (Ed.). Dental Implant Prosthetics. San Luís, Elsevier Mosby, 2005. pp.472-510.
Misch, C. E.; Wang, H. L.; Misch, C. M.; Sharawy, M.; Lemons, J. \& Judy, K. W. Rationale for the application of immediate load in implant dentistry: Part I. Implant Dent., 13(3):207$17,2004$.

Parel, S. M. Immediate implant loading: A review and introduction of a unique new concept. Texas Dent. J., 118(5):364-7, 2001.

Parr, G. R.; Steflik, D. E. \& Sisk, A. L. Histomorphometric and histologic observations of bone healing around immediate implants in dogs. Int. J. Oral Maxillofac. Implants, 8(5):534-40, 1993.

Taylor, T. D.; Agar, J. R. \& Vogiatzi, T. Implant prosthodontics: current perspective and future directions. Int. J. Oral Maxillofac. Implants, 15(1):66-75, 2000.

van Steenberghe, D.; Naert, I.; Andersson, M.; Brajnovic, I.; Van Cleynenbreugel, J. \& Suetens, P. A custom template and definitive prosthesis allowing immediate implant loading in the maxilla: a clinical report. Int. J. Oral Maxillofac. Implants, 17(5):663-70, 2002.

Widmann, G. \& Bale, R. J. Accuracy in computer-aided implant surgery--a review. Int. J. Oral Maxillofac. Implants, 21(2):305-13, 2006.

Wood, M. R.; Vermilyea, S. G. \& Committee on Research in Fixed Prosthodontics of the Academy of Fixed Prosthodontics. A review of selected dental literature on evidence-based treatment planning for dental implants: report of the Committee on Research in Fixed Prosthodontics of the Academy of Fixed Prosthodontics. J. Prosthet. Dent., 92(5):447-62, 2004.

Dirección para Correspondencia:

Yerko Leighton Fuentealba

Facultad de Odontología

Universidad de Chile

Santiago

CHILE

Email: yerkoleighton@gmail.com

Recibido : 05-03-2013

Aceptado: 17-05-2013 